S. Maekawa T. Tohyama S.E. Barnes

S. Ishihara W. Koshibae G. Khaliullin

\title{
Physics \\ of Transition Metal Oxides
}

With 137 Figures 


\section{Contents}

1. Introduction

S. A laekawa $\ldots \ldots \ldots \ldots \ldots \ldots \ldots$
1.1 Transition Metal Ions in Crystal Fields
1.2 Crystal Structure and Physical Properties

2. Cuprates

T. Tohyarna ….............................................................................................. 37

2.1 Underlying Electronic Structure of Cuprates ......................... 38

2.1.1 Charge-Transfer-Type Insulators ................................ 38

2.1.2 Model Hanültonian ........................................................ 41

2.1.3 Superexchange Interaction ............................................ 43

2.1.4 Cyclic Four-Spin Interaction ...................................... 49

2.1.5 Zhang-Rice Singlet State ............................................ 50

2.1.6 Optical Excitations ....................................................... 55

2.2 One-Dimensional Cuprates ...................................................... 58

2.2.1 Spin-Charge Separation ................................................ 58

2.2.2 Realization of Spin-Charge Separation ........................ 63

2.2.3 Charge Dynamits in Insulating Cuprates ..................... 68

2.2.4 Nonlinear Optical Response ............................................. 74

2.2.5 Spin Dynamits in Insulating Cuprates ........................ 79

2.3 Two-Dimensional Cuprates ...................................................... 80

2.3.1 Single Carrier in Mott Insulator ................................... 81

2.3.2 Phase Diagram ................................................................ 86

2.3.3 Optical Conductivity ..................................................... 90

2.3.4 Single-Particle Spectral Function .................................. 93

2.3.5 Chemical Potential ......................................................... 94

2.4 Summary ................................................................................... 95

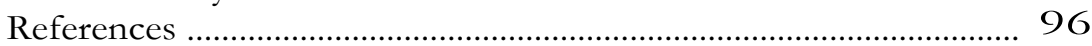


3. Theory of Superconductivity

S.E. Barnes ……......................................................................... 101

3.1 The BCS Pairing Theory ………………................................... 103

3.2 Phonons in Solids ............................................................... 107

3.3 Phonons as Intermediate Bosons................................................. 108

3.4 Theory of the Anti-ferromagnetic Parent Compounds ..................................................................... 111

3.5 The Jordan-Wigner Transformation arid Flux Tubes .............. 116

3.6 Coherent States, Grassman Variables and Flux Tubes ............ 120

3.7 Mean-Field Approximations and .Flux States ............................ 122

3.8 Bogoliubov Theory for a Bose Superfluid .................................. 124

3.9 Auxiliary Particle Methods ............................................................ 126

3.10 Magnetic Exchange Interactions via Intermediate Bosons 130

3.11 The (Mean-Field) RVB Slave Boson Theory ............................ 131

3.12 The Gutzwiller Projection and a U(1) Symmetry .................... 136

3.13 Auxiliary Particles and the Introduction of Flux Tubes 137

3.14 Spin Pa.iring ........................................................................ 140

3.15 Fermionic Excitations in an Anti-ferromagnet .......................... 141

3.16 SU(3) Approach to Hole Coherent States ..................................... 143

3.17 The Effective Exchange for Coherent Doping ………………..... 147

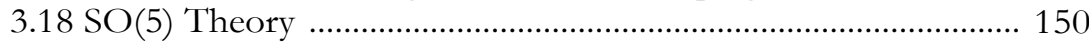

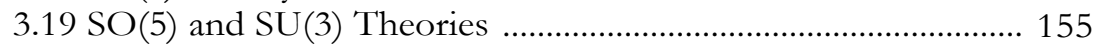

3.20 Gossanier Superconductivity ..................................................... 158

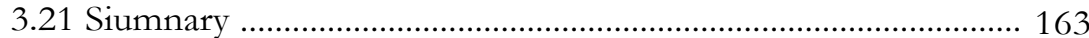

References.................................................................................. 165

\section{Manganites}

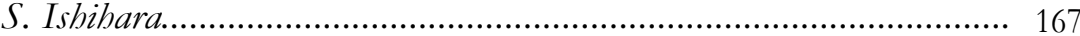

4.1 Manganite with Cubic Perovskite Structure................................ 167

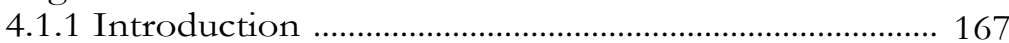

4.1.2 Electronic Hamiltonian and Exchange Interaction ...... 170

4.1.3 jahn-Teller Effect and Cooperative Jahn-Teller Effect 178

4.1.4 Phase Diagram and Orbital Order .................................. 182

4.1.5 Orbital Liquid State ......................................................... 186

4.2 Manganite with Layered Structure .................................................. 190

4.2.1 Phase Diagram ............................................................... 190

4.2.2 Stability of Orbital and Magnetic Structure .................. 193

4.2.3 Experiments for Spin and Orbital Correlation .............. 194

4.3 Resonant X-ray Scattering (RXS) …………............................. 197

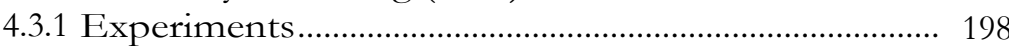

4.3.2 Scattering Cross Section ................................................... 200

4.3.3 Azimuthal Angle Dependence.......................................... 203

4.3.4 Mechanism of RXS ...................................................... 206

4.3.5 Microscopic Calculations of the RXS Intensity ............. 209

4.4 Orbital Excitation .......................................................................... 211 
4.5 Other Orbital-Related Topics ................................................ 216

4.6 Summary .................................................................................... 219

References ................................................................................. 219

5. Titanates and Vanadates

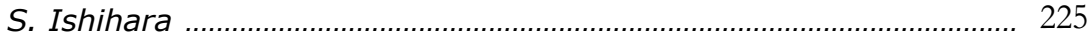

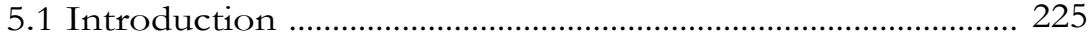

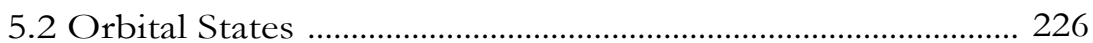

5.2.1 Perovskite Titanates ........................................................ 226

5.2.2 Perovskite Vanadates....................................................... 229

5.3 Metar-Insulator Transition ......................................................... 230

5.4 Electronic State and Model Hamiltonian .................................. 231

5.5 Summary ............................................................................ 238

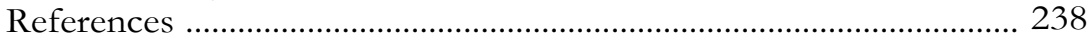

6. Cobaltates

W. Koshibae ……............................................................................. 241

6.1 Low-, High- and Intermediate-Spin States ............................. 241

6.2 Thermoelectric Materials and Cobalt Oxides .......................... 245

6.3 Thermoelectric Effect ................................................................... 246

6.4 Linear Response Theory for Thermoelectric Systems .............. 250

6.5 Thermopower in Strongly Correlated Systems:

Approach from High Temperature Side...................................... 252

6.6 Spin and Orbital States and the Thermopower ....................... 254

6.7 Thermopower of the Degenerate Electron Gas ........................ 257

6.8 Summary and Discussion - 257

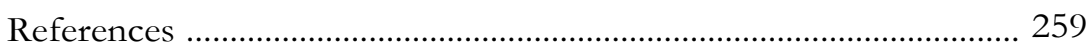

7. Quantum Effects in Orbitally Degenerate Systems

G. Khaliullin ................................................................................ 261

7.1 Systems with $e_{g}$ Orbital Degeneracy .......................................... 262

7.1.1 Spin-Orbital Model ....................................................... 262

7.1.2 Orbital-Only Model .......................................................... 269

7.1.3 Orbital-Charge Coupling, Orbital Polarons ................. 272

7.1.4 Orbital Liquids, Anomalous Transport ........................ 279

7.2 Systems with t $2{ }_{9}$ Orbital Degeneracy ......................................... 282

7.2.1 Spin-Orbital Model ........................................................... 283

7.2.2 Orbital-Only Model .......................................................... 291

7.3 High Spin Systems with $t 2_{q}$ Orbital Degeneracy ..................... 298

7.3.1 Formation of One-Dimensional Orbital Chains ........... 298

7.3.2 Spin-Orbital Dimerization .......................................... 302

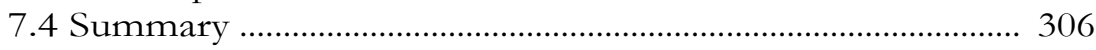

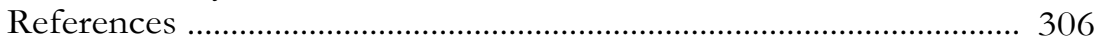


X Contents

Appendices

A. Optical Conductivity

T. Tohyama

B. Exact Diagonalization Technique: The Lanczos Method

T. Tohyama

C. Projection Method (Memory Function Method, Composite Operator Method)

S. Ishihara

D. Thermoelectric Effects

Koshibae

D.1 Seebeck Effect ..................................................................... 323

D. 2 Peltier Effect ........................................................................ 325

D. 3 Thomson Effect .................................................................... 326

D.4 The First and Second Laws of Thermodynamics and Thermoelectric Response in Solids:

Thomson's Considerations

D.5 The Third Law of Thermodynamics and Thermoelectric Response in Solids

References for Appendices

Index 